The basic reproductive ratio as a link between acquisition and change in phonotactics Andreas Baumann \& Nikolaus Ritt

University of Vienna

Andreas Baumann (corresponding author)

Department of English

University of Vienna

Spitalgasse 2-4, Hof 8.3

A-1090 Vienna

Austria

T: +43 $14277-424-27$

Email: andreas.baumann@univie.ac.at

Nikolaus Ritt

Department of English

University of Vienna

Spitalgasse 2-4, Hof 8.3

A-1090 Vienna

Austria

T: +43 1 4277-424-24

Email: nikolaus.ritt@univie.ac.at 


\title{
The basic reproductive ratio as a link between acquisition and change in phonotactics
}

\begin{abstract}
1 Language acquisition and change are thought to be causally connected. We demonstrate a

2 method for quantifying the strength of this connection in terms of the 'basic reproductive

3 ratio' of linguistic constituents. It represents a standardized measure of reproductive success,

4 which can be derived both from diachronic and from acquisition data. By analyzing English

5 data, we show that the results of both types of derivation correlate, so that phonotactic

6 acquisition indeed predicts phonotactic change, and vice versa. After drawing that general

7 conclusion, we discuss the role of utterance frequency and show that the latter only exhibits

8 destabilizing effects on late acquired items, which belong to phonotactic periphery. We

9 conclude that - at least in the evolution of English phonotactics - acquisition serves

10 conservation, while innovation is more likely to occur in adult speech and affects items that

11 are less entrenched but comparably frequent.
\end{abstract}

12

13

14 Keywords: diachronic linguistics, language acquisition, reproductive success, basic

15 reproductive ratio, phonotactics, dynamical systems

16

17 

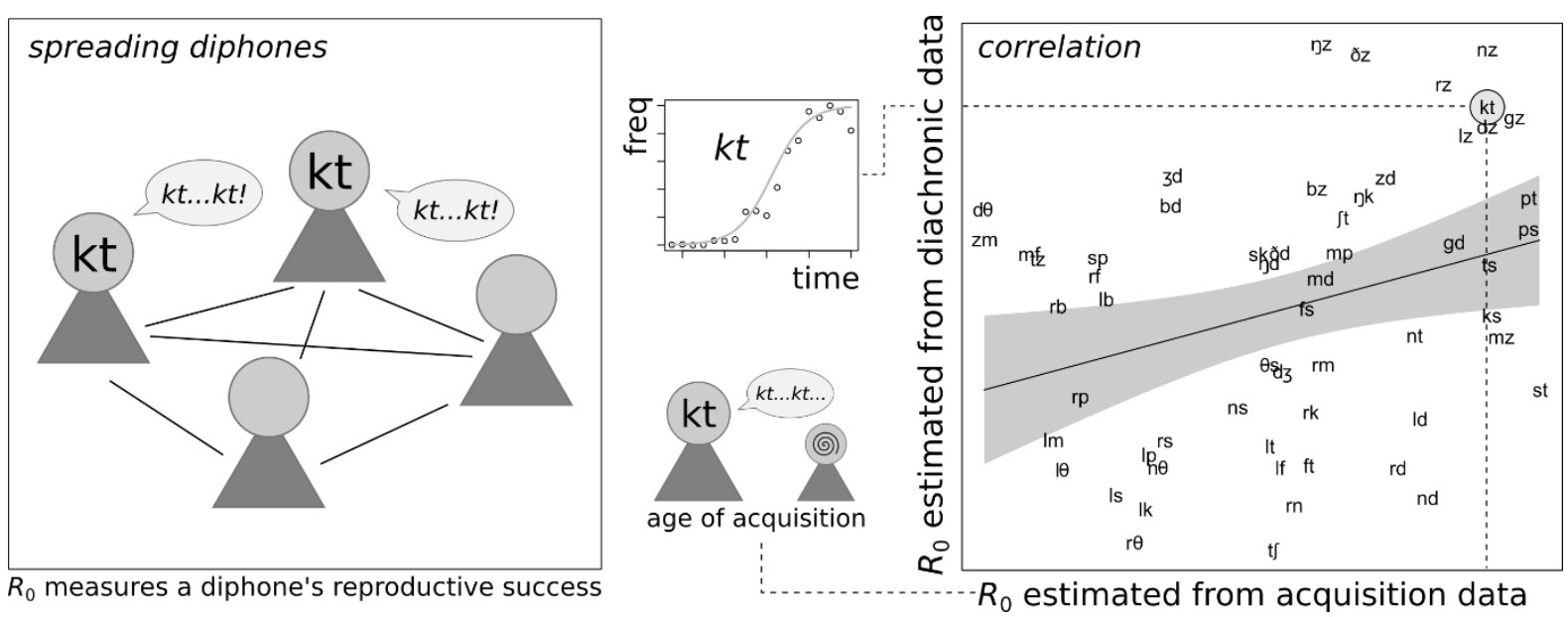

$21 \quad 1 \quad$ Introduction

22 Languages are systems of mental instructions that are shared by their speakers. They are instantiated in the mind-brains of many individuals and transmitted across generations through communicative interaction and language acquisition. For a constituent of linguistic knowledge to be successfully transmitted across generations, it needs to be used and expressed by adult speakers in such a way that new generations can acquire it successfully.

27 Thus, the history of language constituents depends on language use and language acquisition and is likely to reflect constraints on both of them. This paper focusses on the relation between history and acquisition.

That language acquisition is crucial for language history is trivially true and generally acknowledged (Briscoe, 2008; Smith \& Kirby, 2008). After all, constituents that are not acquired cannot survive. However, the matter is both more complex and more interesting than that. On the one hand, there is considerable disagreement about how much language

34 acquisition contributes to linguistic change, and on the other hand, some correlations between acquisition and diachronic stability appear to be quite specific. For instance, Monaghan 
36 (2014), demonstrates that the age at which a lexical item is acquired predicts the diachronic stability of its phonological form. The finding has inspired various attempts to account for it, but no consensus has been reached. On one interpretation, early acquisition is thought to cause diachronic stability: early acquired items become strongly entrenched, get to be used frequently, and are therefore more likely to be historically stable than items that are acquired later (MacNeilage \& Davis, 2000; Monaghan, 2014). On another view, early acquisition and diachronic stability are thought to have common causes: items will both be acquired early and remain diachronically stable if they are easily produced, perceived, or memorized, for example.

This paper explores the relation between the diachronic stability of linguistic constituents and the age at which they are acquired. To determine how systematic that relation is, we introduce and test a rigorous quantitative model that relates patterns attested in historical language development to patterns attested in language acquisition. More specifically, we show how age-of-acquisition and diachronic stability can be related to each other in terms of

50 a standardized measure of reproductive success, namely their 'basic reproductive ratio'

51 (henceforth $R_{0}$ ) (Dietz, 1993; Heffernan, Smith, \& Wahl, 2005). That measure (more on it below, see 2.1) has proved useful in the study of population-dynamics. We use a population dynamic model ${ }^{1}$ that has already been applied to explain linguistic phenomena (Nowak, 2000; Nowak, Plotkin, \& Jansen, 2000) and show in which way estimates of $R_{0}$ can be derived for linguistic constituents. Crucially, they can be derived both from age-ofacquisition data and from diachronic corpus evidence. By comparing the two estimates, one

\footnotetext{
${ }^{1}$ That model we use is similar to mathematical models of cultural and linguistic change (Cavalli-Sforza and Feldman (1981); Wang and Minett (2005); Niyogi (2006)) and equivalent to basic epidemiological models (Anderson and May (1991); see also Sperber (1985)).
} 
57 can then put numbers on the relation between language acquisition and language history. Thus, the model provides a method for relating data of different origins mechanistically. Empirically, our discussion is based on English word-final CC diphones (i.e. consonant clusters containing two segments). They are short, yet clearly structured linguistic constituents (Kuperman, Ernestus, \& Baayen, 2008), and have had long and diverse histories. For instance, the word final cluster /nd/ as in English land is likely to have existed already more than 5000 years ago in Indo-European, the ancestor of English. It still thrives today. Many others, however, such as /gz/ or /vz/ as in English legs or loves, emerged much more recently, i.e. about 800 ago in the Middle English period. There are also considerable differences among the histories of individual clusters as far as their frequencies are concerned. Some of them, such as /xt/ - graphically still reflected in words like knight or laughed - have disappeared altogether.

Since (a) there is considerable diversity among the historical developments of final consonant clusters, and since (b) the ages at which they are acquired are similarly diverse, English consonant clusters are highly suitable for our purpose. They allow us to see clearly whether the reproductive ratios that population dynamic models derive from historical evidence and acquisition data actually correlate or not. We show that they do and interpret this as proof of the concept that models which derive $R_{0}$ for linguistic constituents are capable of relating language acquisition and language history in a meaningful way. Thus - and although we are interested in the specific phenomena we investigate our primary concern is in fact more general. In the context of testing the usefulness of population dynamic models for linguistic purposes, we address questions such as the following: (a) Does the age at which consonant clusters are acquired correlate with their historical stability? (b) Is there a single measure that relates these two properties? (c) What 
81 can be learnt from such measurements about causal relations between language acquisition and language history?

For (a) and (b), our study suggests positive answers: models developed in the study of evolutionary dynamics do indeed provide systematic and quantifiable correlations between the historical development of final clusters and the age at which are acquired. With regard to (c), we ask if the correlation between acquisition and diachronic stability differs between morpheme internal clusters (such as $/ \mathrm{mp}$ / in lamp) and morphologically produced ones (such as /gz/ in eggs), and whether the correlation between age-of-acquisition and historical stability is affected by utterance frequency. We show that the morphological status of clusters does not seem to matter much, but that the correlation between age-of-acquisition and historical stability is tighter among frequent than among rare clusters. Our results corroborate the view that phonological change may be more strongly driven by frequent use in adult speech (Bybee, 2007), and that early acquired core items are more resistant against frequency-driven effects like reduction, assimilation, or deletion. Thereby, our study contributes to the debate on the role which language acquisition plays in language change.

In terms of its general approach, our paper relates to a growing body of research that views culturally transmitted knowledge in evolutionary terms and models it accordingly (Cavalli-Sforza \& Feldman, 1981; Dawkins, 1976; Henrich \& Boyd, 2002; Newberry, Ahern, Clark, \& Plotkin, 2017). It is also based on the view that the repeated learning events 100 involved in cultural history can amplify and make visible cognitive biases that are too weak 101 to be traceable in the behavior of individuals (Reali \& Griffiths, 2009; Smith et al., 2017; Smith \& Wonnacott, 2010).

We describe our modeling approach together with both ways of estimating the basic 104 reproductive ratio in Section 2. After that, we introduce the statistical tools (3) which are used 
105 to empirically test our model against data from phonotactic acquisition and diachrony. The 106 results of our analysis (4) are finally discussed in Sections 5 and 6, thereby particularly

107 focusing on the effect of utterance frequency.

\section{Data and methods}

\subsection{Standardizing reproductive success: basic reproductive ratio}

110 Our analysis employs a modified version of the population dynamical model of linguistic

111 spread proposed by Nowak and colleagues (Nowak, 2000; Nowak et al., 2000; Solé, 2011).

112 For each linguistic constituent, i.e. in our case for each cluster, the model consists of two

113 differential equations that track the growth of the number of 'users' $U$ (speakers that know

114 and use the cluster), and the number of 'learners' $L$ that do not (yet) know or use it.

115 When users and learners meet, learners acquire the cluster at a rate $\alpha>0$, whereby

116 they become users (i.e. switch from class $L$ to class $U$ ). Conversely, at a rate $\gamma=1 / G$, where

$117 G>0$ is linguistic generation time, users 'die' (i.e. are removed from class $U$ ) and learners

118 are 'born' (i.e. added to class $L$ ). The respective rates of change thus read

$$
\begin{aligned}
\dot{L} & =-\alpha L U+\gamma U \\
\dot{U} & =\alpha L U-\gamma U
\end{aligned}
$$

119 where we set $L+U=1 .^{2}$

120 The expected number of learners that acquire a cluster from a single user introduced

121 into a population of learners is $R_{0}=\alpha / \gamma$ (Hethcote, 1989). $R_{0}$ represents what has been

122 labelled 'basic reproductive ratio' (Anderson \& May, 1991; Nowak, 2000). It figures

\footnotetext{
${ }^{2}$ For $\gamma=1$, the above system is exactly the model of word dynamics in Nowak (2000). In his model, $\alpha$ depends on the utterance frequency and learnability of a word, as well as on the number of informants a learner is exposed to (network density).
} 
123 centrally in epidemiological research due to its straightforward properties: whenever it holds

124 for a population (e.g. a subpopulation of infected individuals) that $R_{0}>1$, that population

125 increases in size and spreads.

126 In our model, $R_{0}>1$ entails that the population of users approaches a stable

127 equilibrium $\widehat{U}=1-\gamma / \alpha=1-1 / R_{0}$, so that $\widehat{L}=1 / R_{0}$. If, on the other hand, $R_{0}<1$, the

128 fraction of users approaches 0 . The linguistic item vanishes. $R_{0}$ represents a standardized measure of reproductive success that reflects the

130 diachronic stability of linguistic items. Its greatest asset is that it can be derived from

131 different types of data and that all derived estimates are situated on the same scale. Thus,

132 estimates derived from different data types can be compared directly and without further

133 transformation. In our paper, we exploit this for comparing the $R_{0}$ derived from diachronic

134 frequency data to the $R_{0}$ derived from language-acquisition data. We show that such a

135 comparison yields interesting perspectives on the relation between age of acquisition and 136 historical stability.

\section{$137 \quad 2.2 \quad$ Estimating reproductive success from diachronic growth}

138 The model of linguistic spread outlined in the previous section can be reformulated in terms

139 of a logistic equation (Hethcote, 1989; Solé, Corominas-Murtra, \& Fortuny, 2010) with an

140 intrinsic (potentially negative) growth rate $\rho=\alpha-\gamma$. Thus, if the linguistic generation time

$141 \mid G:=1 / \gamma$ and the growth rate $\rho$ are known, then $\alpha$ and $\alpha / \gamma=1+\rho G=: R_{0}^{\mathrm{GR}}$ can be

142 determined. We approximate $G$, i.e. the average time it takes for new language learners to

143 enter the population, by biological generation time, so that $G \cong 30$ years (Worden, 2008).

144 This leaves the intrinsic growth rate $\rho$ to be determined. 
In order to estimate the intrinsic growth rates $\rho$ of final CC clusters, we use logistic

146 growth rates $r_{\mathrm{lg}}$ obtained from diachronic frequency data as a proxy (see also the discussion

147 in section 5). For that purpose, we determine a trajectory of normalized token frequencies $f$

148 from 1150 to 2012 for each word-final CC cluster. The token frequencies were retrieved from

149 various historical and contemporary language databases and corpora (see Table 1, which also

150 indicates who carried out the phonological interpretation). The collected data were divided

151 into periods of 50 years, yielding 18 data points for each final $\mathrm{CC}$ cluster.

152

153 Table 1. Diachronic data covering the lineage from Early Middle English to Contemporary

154 American English. Data were binned into periods of 50 years each (e.g. 1200 denoting 1200-

1551250 below). In the case of overlapping data sets (e.g. PPCMBE2 and COHA in the $19^{\text {th }}$

156 century) weighted averages based on both corpus sizes were used to compute frequencies.

157 Since we trace the American English lineage (COHA, COCA), phonological transcriptions

158 for the late periods were taken from CMPD.

\begin{tabular}{l|l|l} 
Sources for frequencies & Covered periods & Phonological interpretation \\
\hline PPCME2 (Kroch \& Taylor, 2000) & $1150,1200, \ldots, 1450$ & [Authors $]$ \\
\hline PPCEME (Kroch, Santorini, \& Delfs, & $1500,1550, \ldots, 1700$ & \\
2004) & & \multirow{2}{*}{ CMPD (Carnegie Mellon } \\
\hline PPCMBE2 (Kroch, Santorini, \& & $1700,1750, \ldots, 1900$ & Speech Group, 2014) \\
Diertani, 2016) & &
\end{tabular}

159 
We chose 1150 to 2012 as our observation period because word final CC clusters were rare before (i.e. in Old English). The vast majority of them was only first produced by schwa loss in final syllables, which started roughly at this time (Minkova, 1991). Note that although the phonological process of schwa loss affected word final sequences quite uniformly in the early Middle English period, the different cluster types it produced developed relatively independently of each other after schwa loss was completed (in the $15^{\text {th }}$ century). This reflects the post-medieval influx of loans ending in CC clusters as well as phonological processes other than schwa loss - for instance final devoicing - that produced new clusters. For most of the observation period the dynamics of the individual cluster types can thus be considered as relatively independent from each other. The derived trajectories were normalized to the unit interval with respect to their maximum values, and subsequently fit to a logistic model given by $f(t)=1 /(1+$ $\left.\exp \left(-r_{\lg }\left(t-t_{0}\right)\right)\right)$, where $t_{0}$ was set at the middle of the observation period. Non-linear

174 least-squares regression was used to estimate $r_{\text {lg }}$ for each cluster. The quality of this estimate depends on the actual shape of the empirical trajectory. Since the model presupposes

176 (positively or negatively) unidirectional development, $r_{\mathrm{lg}}$ estimates can be unreliable for clusters who show (inverse) U-shaped developments. Therefore, we also computed Spearman's Rho $\left(\mathrm{P}_{\mathrm{sp}}\right)$ for each cluster. We excluded clusters for which $\left|\mathrm{P}_{\mathrm{sp}}\right|$ scored below the

179 threshold of 0.1 , to rule out clearly non-monotonous developments. ${ }^{3}$ This also eliminated clusters that occurred only sporadically in a few periods. Finally, we did not consider final

\footnotetext{
${ }^{3}$ We are grateful to an anonymous reviewer for addressing the issue of non-monotonous patterns. The employed threshold $\left|\mathrm{P}_{\mathrm{sp}}\right|>0.1$ is relatively mild, as we wanted to keep our data set reasonably large. It excludes only trajectories that are strongly non- monotonous. The qualitative results of this paper still apply up to a threshold of $\left|\mathrm{P}_{\mathrm{sp}}\right| \sim 0.3$.
} 
181 cluster types that are absent in Present Day English such as $/ \mathrm{mb}$ / in limb because there are no

182 data on the age at which they are acquired. Thus, a total of 58 final CC types entered our

183 analysis (Table A1 in the appendix). For the purpose of illustration, Figure 1 shows logistic

184 models for nine different cluster types: for instance, /kt/ exhibits a sigmoid increase in

185 frequency (i.e. $r_{\mathrm{lg}}>0$ and $R_{0}^{\mathrm{GR}}>1$ ), while $/ \mathrm{rn} /$ becomes less frequent $\left(r_{\mathrm{lg}}<0\right.$ and $R_{0}^{\mathrm{GR}}<$ 186 1).

187
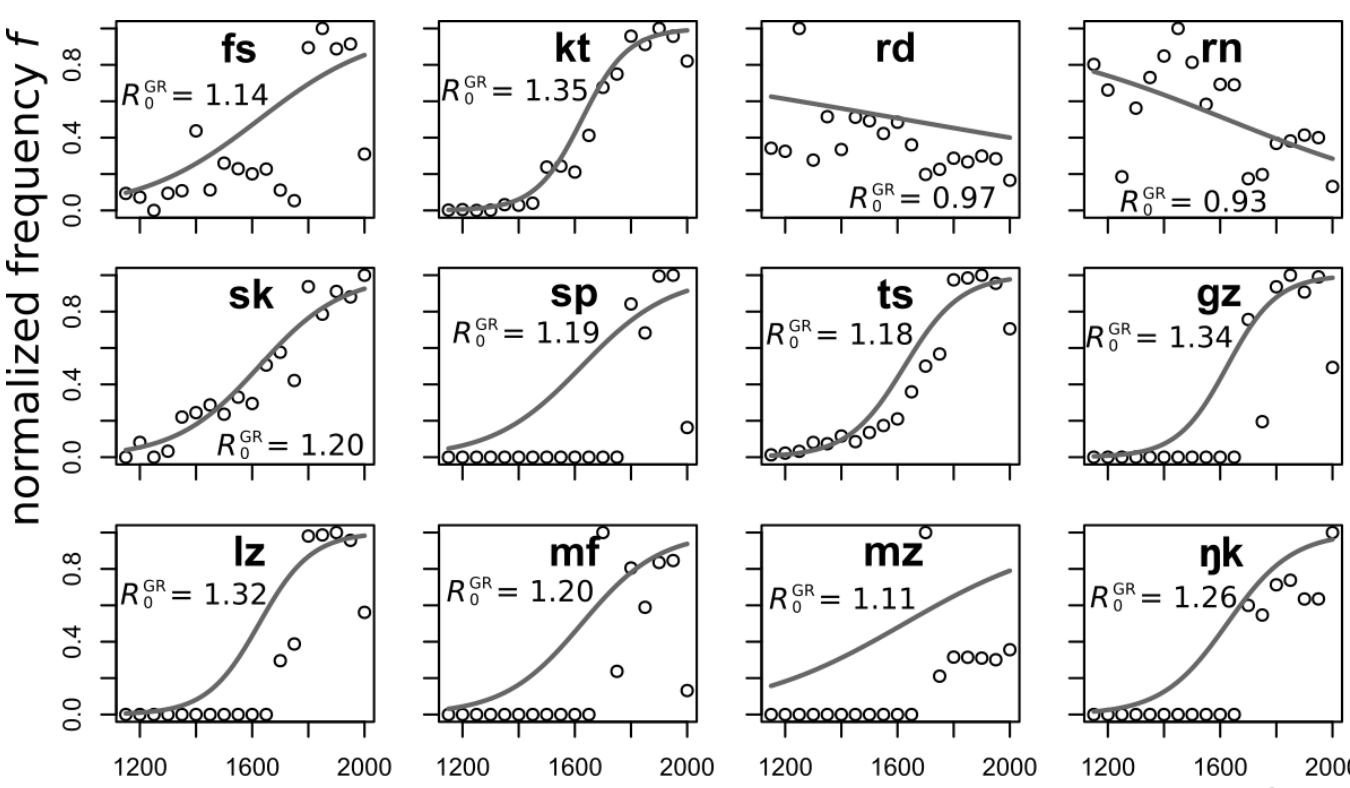

188
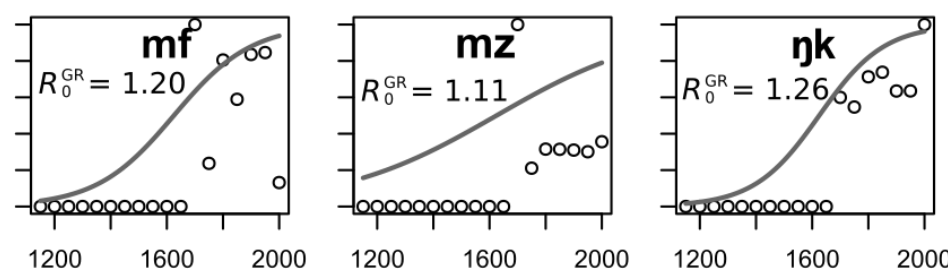

time

189 Figure 1. Logistic growth curves for a set of English word-final CC-clusters. All clusters

190 show a non-trivial monotonous development (decreasing or increasing). The graphs were

191 selected in order to represent a large variety of diachronic patterns. In some cases (e.g. /sk/,

192 /ts/, /sk/) trajectories fit the logistic pattern remarkably well. In other cases (e.g. /rn/, /fs/, /sp/)

193 they don't. Some clusters feature extremely low frequencies in early periods. 


\section{5 \\ 2.3 Estimating reproductive success from age of acquisition}

196 Next, we derived $R_{0}$ estimates from language acquisition data. Here, our derivation follows

197 Dietz (1993). The population of linguistic agents is once again split into a fraction $L$ of

198 'learners' and a fraction $U$ of 'users' for each linguistic item. AoA denotes the age of

199 acquisition of that item and LE denotes the life expectancy of an individual. Under the

200 assumption of a roughly rectangular age structure (Dietz 1993), at equilibrium LE/AoA =

$201(\widehat{L}+\widehat{U}) / \widehat{L}=R_{0}=: R_{0}^{\text {AoA }}$. It is therefore sufficient to estimate AoA, as long as LE is known.

202 For the sake of simplicity, we assume a constant life-expectancy of LE $\cong 60$ years

203 (Lancaster, 1990: 8). ${ }^{4}$

Our estimates for the AoAs of 58 final clusters are based on Kuperman et al.'s

205

206

207

208

209

210

211

212

213

(2012) AoA ratings for 30,000 English words. These ratings were collected in a broad

crowdsourcing study among speakers of American English and correlate highly with ratings obtained under laboratory conditions (see also Monaghan 2014). The AoA of a cluster type was operationalized as the mean of the AoA ratings of the three earliest-acquired word-forms containing it. Averaging over the first three acquired items containing a cluster yields a more robust measure of its AoA than considering only the very earliest word containing it. Since we treat $\mathrm{CC}$ clusters as linguistic constituents in their own right (and not just as properties of words), we consider their acquisition to require exposure to more than a single word containing them. Nevertheless, we operationalize the AoA of a cluster as a point estimate that

\footnotetext{
${ }^{4}$ Note that the results presented in Section 4 are qualitatively robust with respect to altering life expectancy since $R_{0}^{\mathrm{AoA}}$ scales linearly with LE. Nevertheless, incorporating time dependent LE would represent an interesting but substantially more complex extension of our method.
} 
214 divides the life of a speaker into a period before and a period after acquisition of that cluster

215 (i.e. the transition date from $L$ to $U) .^{5}$

216 Word-forms in which final CC clusters result from morphological operations (such

217 as /gz/ in the plural egg $+s$ ) received the AoA rating of the base forms contained the data set

218 (e.g. egg). There are two reasons why this is likely to yield plausible estimates. First, the

219 lowest AoA rating in our data is 2.74, and the majority of English inflectional morphology is

220 acquired during between 2.25 to 3.75 years (Brown, 1973). Furthermore, it has been shown

221 that in languages which are morphologically poor (such as English as opposed to Polish)

222 there is no significant difference between the ages at which morphologically produced and

223 morpheme-internal clusters are acquired (Korecky-Kröll et al., 2014, p. 48). Transcriptions

224 were once again taken from CMPD.

\section{$225 \quad 2.4 \quad$ Utterance frequency}

226 Frequency has often been argued to affect the diachronic stability of linguistic items (Bybee

227 2007). Thus, Pagel et al. (2007) show that the rate of phonological change in the lexicon can

228 be predicted from the frequency of word use. At the same time, frequent words are acquired

229 earlier than rare ones (Kuperman et al. 2012). This suggests that frequency increases

230 reproductive success. On the other hand, utterance frequency has also been shown to drive

231 phonological erosion. Frequent words are also comparably expectable and therefore more

232 tolerant of reduction (Bybee \& Hopper 2001; Diessel 2007). Thus, it is unclear if frequency

233 should increase or decrease the diachronic stability of CC clusters.

\footnotetext{
${ }^{5}$ This operationalization of AoA is most compatible with the underlying population dynamical model. We found that the exact operationalization of AoA is crucial to the comparison of the two derived $R_{0}$ estimates. AoA ratings for clusters that are derived from the AoAs of all words containing it get implausibly high because some of those words are inevitably acquired extremely late and unlikely to play any role in the acquisition of a cluster.
} 
In order to investigate that issue, our study takes frequency into consideration as an

additional factor. Since cluster-specific utterance frequencies fluctuate during the observation period, we first extracted per million normalized token frequencies for all cluster types in every single period of 50 years. In addition, we computed average token frequencies for each cluster type across all 18 periods, denoted as 〈frequency $\rangle$ in order to obtain a more compact summary measure (see Table A1 in the appendix).

\subsection{Morphology}

241 While syntax or pragmatics have little immediate influence on word internal phonotactics, morphology affects it strongly. Thus, many word-final CC clusters result from morphological operations (Dressler, Dziubalska-Kołaczyk, \& Pestal, 2010; Hay \& Baayen, 2005). As far as

244 the acquisition of morpheme-internal phonotactics is concerned, however, we do not expect morphology to contribute much (see 2.3). In our observation period, English syntheticity (i.e. the amount of morphological operations) underwent a non-uniform development which exhibits a U-shaped curve, as demonstrated by Szmrecsanyi (2012). Thus, the interaction of morphology and the diachronic dynamics of word-final phonotactics is a priori not so clear.

249 In order to account for morphological effects in our analysis, we classified final CC types as

250 (a) (exclusively) morphologically produced (and 'illegal' within morphemes, e.g. /md/ in seemed), (b) (exclusively) morpheme internal ('legal', /lp/ in help), or (c) both ('mixed', /nd/ in hand and planned).

\section{Calculation}

254 To explore the relative impact and the interaction of the different factors, we employed linear 255 models (LM) and generalized additive models (GAM, Wood, 2006a). First, z-normalized 
256 estimates of $R_{0}^{\mathrm{GR}}$ (the reproductive ratio derived from diachronic growth data) and $R_{0}^{\mathrm{AoA}}$ (the

257 reproductive ratio derived from age-of-acquisition data) entered a LM as dependent and

258 independent variables (Model 1a). No transformation (e.g. log) was needed for either

259 variable. The effect of morphology ('illegal'; 'mixed'; 'legal'; the latter as default) was

260 analyzed by adding a linear interaction term to the previous model (Model 1b).

Analyzing the interaction of frequency with the derived $R_{0}$ measures is more

complicated because it involves time as an additional factor. Initially (Model 2), normalized

(i.e. z-transformed) log-transformed average frequency, 〈frequency $\rangle$, was integrated as an

interacting variable into a GAM, in which $R_{0}^{\mathrm{AoA}}$ figures as predictor and $R_{0}^{\mathrm{GR}}$ as dependent

variable. The interaction between $R_{0}^{\mathrm{AoA}}$ and logged 〈frequency) was modeled by means of a tensor-product term (Wood, 2006b). The effects of logged 〈frequency) on $R_{0}^{\mathrm{GR}}$ and $R_{0}^{\mathrm{AoA}}$ were then evaluated in two separate GAMs (Model 3a and 3b, respectively). In both of them, logged 〈frequency $\rangle$ figures as predictor (smooth term). Finally, the interaction of time and

269 logged frequency - both affecting $R_{0}^{\mathrm{GR}}$ and $R_{0}^{\mathrm{AoA}}$ respectively -, was modeled as a tensor

270 product term in two additional GAMs (model $4 \mathrm{a}$ and $4 \mathrm{~b}$, respectively). ${ }^{6}$

\section{Results}

272 The direct comparison of the two estimates of $R_{0}$ (model 1a, Fig. 2) reveals a non-trivial

273 linear relationship between the two variables (standardized coefficient $\beta_{\mathrm{AoA}}=0.31 \pm$

$2740.13 S E$ at $p=0.016$ ). Adding morphology (model $1 \mathrm{~b}$ ) does not reveal a statistically

275 significant interaction and decreases the explanatory power of the model $\left(\beta_{\text {AoA }}=0.20 \pm\right.$

\footnotetext{
${ }^{6}$ All models based on Gaussian distribution with identity link. The number of knots in smooth terms was deliberately kept low in order to detect monotone and easy to interpret (but still possibly nonlinear) relationships.
} 
$\left.2760.23 S E ; \beta_{\text {AoA } \times \text { mixed }}=-0.04 \pm 0.33 S E ; \beta_{\text {AoA } \times \text { illegal }}=0.48 \pm 0.37 S E\right){ }^{7}$ Thus, we can

277 assume the discovered correlation to hold irrespective of morphological status.

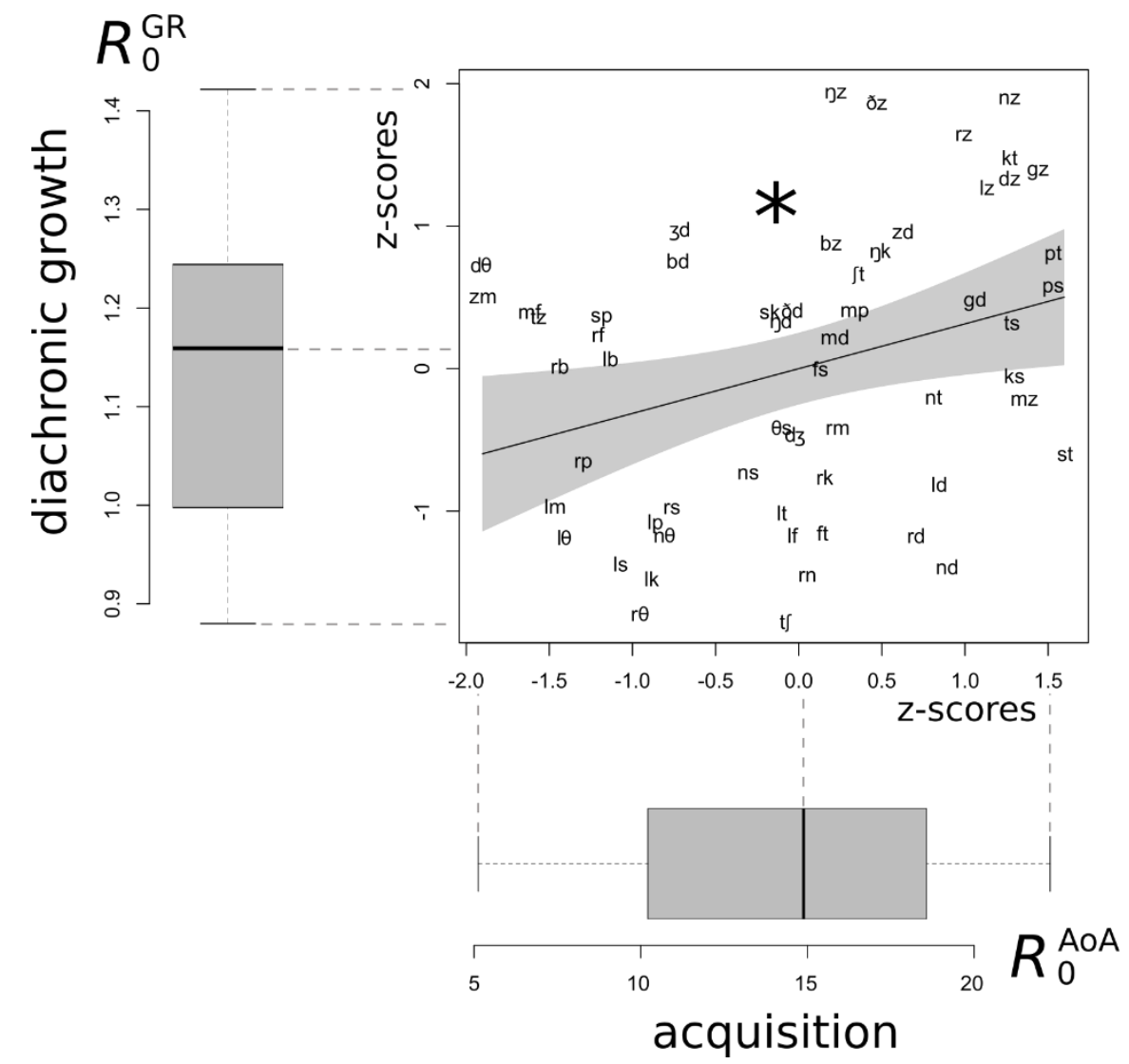

279 Figure 2. Linear relationship between normalized estimates of $R_{0}^{\mathrm{GR}}$ (vertical axis) and $R_{0}^{\mathrm{AoA}}$

280 (horizontal axis) (model 1; $p<0.05$ ). Gray areas denote 95\% confidence regions. Boxplots

281 next to the vertical and horizontal axis indicate the distribution of $R_{0}^{\mathrm{GR}}$ and $R_{0}^{\mathrm{AoA}}$,

282 respectively. Scores derived from acquisition data are considerably higher than scores estimated from diachronic data.

\footnotetext{
${ }^{7}$ Model 1a: $R^{2}(\operatorname{adj})=0.08, F=6.13, p=0.016, A I C=163.56 ;$ model $1 \mathrm{~b}: R^{2}(\mathrm{adj})=$ $0.10, F=3.05, p=0.04, \quad A I C=164.5 ;$ model $2: R^{2}(\operatorname{adj})=0.11,16.5 \%$ explained deviance; model 3a: $R^{2}$ (adj) $=0.05,7.00 \%$ explained deviance; model 3b: $R^{2}$ (adj) $=0.36$, $37.5 \%$ explained deviance; model $4 \mathrm{a}: R^{2}(\mathrm{adj})=0.20,20.7 \%$ explained deviance; model $4 \mathrm{~b}: R^{2}(\mathrm{adj})=0.33,34.1 \%$ explained deviance.
} 
Model 2 (Fig. 3a, right) reveals that the relationship between $R_{0}^{\mathrm{GR}}$ and $R_{0}^{\mathrm{AoA}}$,

established in model 1, is much tighter for frequent clusters (e.g. /ns/ as in hence vs. /st/ as in best) than for infrequent ones, where it is approximately constant (/rp/ as in harp vs. /lk/ as in milk; interaction term: $d f=4.33, F=4.76, p<0.001)$. Another way of looking at Fig. 3a is this: in the phonotactic core inventory (i.e. among early acquired clusters), frequency does not affect diachronic stability, while in the phonotactic periphery (among late acquired clusters), frequency reduces it significantly (Fig 3a, left). $d f=1, F=4.20, p=0.045$; linear effect $\left.\beta=-0.24, \mathrm{CI}_{0.95}=(-0.50,-0.01)\right)$. Thus, clusters that have been relatively abundant in the history of English have not become more frequent. ${ }^{8}$ In contrast, model $3 \mathrm{~b}$ (Fig. $3 \mathrm{~b}$ ) shows that $R_{0}^{\text {AoA }}$ positively correlates with average frequency (smooth term: $d f=1, F=33.57, p<0.001$; linear effect $\beta=0.61, \mathrm{CI}_{0.95}=$

(Fig. 3c) shows that frequency and $R_{0}^{\mathrm{GR}}$ were inversely related in the beginning of the observation period but not during more recent periods. The relationship between frequency and $R_{0}^{\text {AoA }}$ (model $4 \mathrm{~b}$, Fig. 3c) was slightly negative in the early part of the observation period but evolved towards a strongly positive interaction later on (interaction term: $d f=4.6$, $F=81.8, p<0.001)$

\footnotetext{
${ }^{8}$ Model 3a was additionally fit to all clusters with $R_{0}^{\mathrm{AoA}}>1$ ('core' items) and $R_{0}^{\mathrm{AoA}}<-1$ ('periphery' items), respectively, in order to make the effect of frequency more clearly visible. Core items: smooth term at $d f=1, F=0.58, p=0.47\left(n=12, R^{2}\right.$ (adj) $=-0.04$, $5.47 \%$ explained deviance). Periphery items: significantly decreasing smooth term at $d f=3.06, F=25.3, p<0.001\left(n=12, R^{2}(\operatorname{adj})=0.90,92.5 \%\right.$ explained deviance $)$.
} 

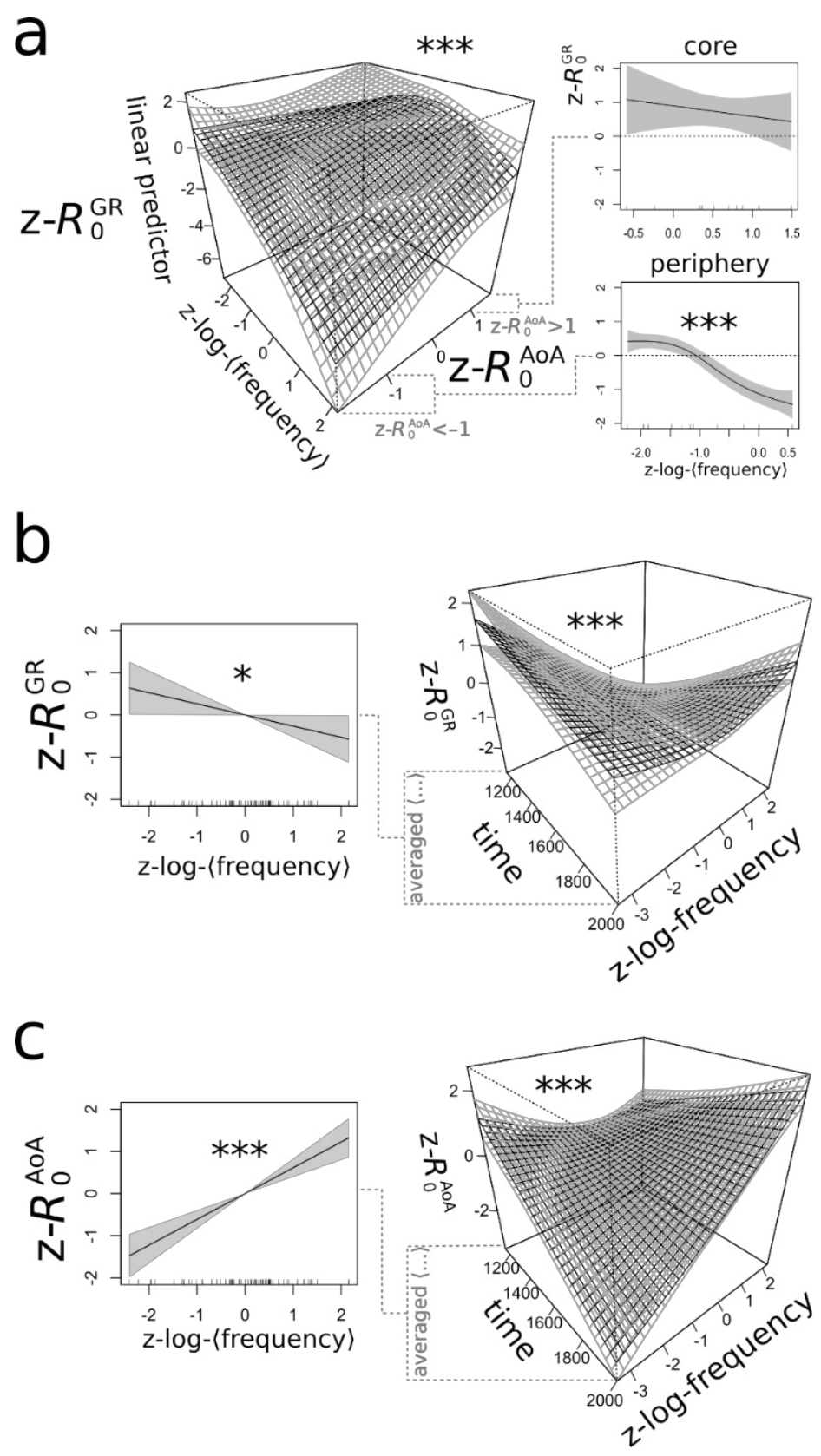

306 Figure 3. (a) Left: The effect of cross-temporally averaged frequency, 〈frequency〉, on the 307 relationship between $R_{0}^{\mathrm{GR}}$ and $R_{0}^{\mathrm{AoA}}$ (z-scores; 〈frequency〉 log-transformed; model 2). The 308 positive relationship becomes stronger as 〈frequency increases and vanishes in low-

309 frequency items. Right: 〈frequency) decreases $R_{0}^{\mathrm{GR}}$ significantly when looking at periphery 
310 items $\left(z-R_{0}^{\mathrm{AoA}}<-1\right)$ but not in the core inventory $\left(z-R_{0}^{\mathrm{AoA}}>1\right)$ (model 3a with restricted

311 data set). (b) Left: 〈frequency〉 decreases $R_{0}^{\mathrm{GR}}$ (model 3a). Right: Frequency (log- and z-

312 transformed) computed for each period of 50 years separately and related with $R_{0}^{\mathrm{GR}}$ and time

313 (model 4a). (c) Left: Same as in (b) with $R_{0}^{\mathrm{GR}}$ replaced by $R_{0}^{\mathrm{AoA}}$, which correlates positively

314 with 〈frequency) (model 3b). Right: Over the past 800 years, a strongly positive relationship

315 between frequency and $R_{0}^{\mathrm{AoA}}$ established itself (model 4b). Recall that $R_{0}^{\mathrm{AoA}}$ is based on

316 contemporary AoA estimates.

317

318

\section{Discussion}

320 We have shown that a simple population-dynamical model of linguistic spread derives

321 correlating estimates of reproductive success from age-of-acquisition data on the one hand, and from diachronic corpus data on the other. At least for English final CC clusters, this means that the basic reproductive ratio ${ }^{9} R_{0}$ qualifies as a standardized measure of reproductive success which allows to the relate AoA with diachronic growth. It has a clear linguistic interpretation and permits the direct comparison of data of various origins (Heffernan et al., 2005).

The correlation between the estimates derived from acquisition data and diachronic evidence supports the widely shared view that age of acquisition and diachronic stability are causally linked. Concurring with Monaghan (2014), our study suggests that what is acquired early is diachronically more stable (and vice versa). Interestingly, however, the tightness of this relationship increases with the frequency of CC clusters. This means that frequent

\footnotetext{
${ }^{9}$ Defined as the expected number of learners that acquire an item from a single user.
} 
332 clusters are not simply acquired before rare ones, but that the historical stability of a cluster

333 can be more confidently predicted from the age at which it is acquired when that cluster is

334 frequent. Among rare clusters the correlation is not as tight. At the same time, these results

335 show that late acquired items from the phonotactic periphery suffer most from frequency

336 driven effects such as assimilation, reduction, or deletion. In that respect, they differ strongly

337 from early acquired - and highly entrenched - core items. Thus, the notion than utterance

338 frequency reduces historical stability still applies (e.g. via erosion in adult speech; Bybee,

339 2007), but we have demonstrated it to be restricted to the periphery.

340 The correlation between frequency and $R_{0}$ estimated from AoA is not surprising. It

341 reflects the way in which the (linguistic version of the) basic reproductive ratio is derived.

342 According to Nowak (2000), $R_{0}$ depends on (a) the ease with which a linguistic item is learnt

343 and memorized, (b) utterance frequency, and (c) the density of the speaker network. Thus,

344 our results highlight the importance learnability for the successful replication of phonotactic

345 items ([Authors]; Croft, 2000; Smith \& Kirby, 2008). In that sense, age of acquisition seems

346 to reflect linguistic and cognitive constraints on the production and the perception of clusters,

347 and on their role in further cognitive processing. These constraints may act on articulatory

348 and perceptual properties of clusters, such as (differences in) the manner or the place of their

349 articulation (Berent, Steriade, Lennertz, \& Vaknin, 2007; Mesgarani, Cheung, Johnson, \&

350 Chang, 2014), or on their semiotic functionality (such as boundary signaling, see McQueen,

351 1998, Dressler et al., 2010).

352 It is interesting that there is no simple positive correlation between $R_{0}$ estimated

353 from historical data and utterance frequency. That would have been expected given the way

354 in which Nowak (2000) defines the basic reproductive ratio. It would also have been

355 expected from previous empirical findings, e.g. by Pagel et al. (2007) or Lieberman et al. 
356 (2007). In fact, taking frequency averaged over the entire observation period into account the opposite seems to be the case, very much in line with the view that high utterance frequency decreases an item's phonological stability (Bybee, 2007, 2010; Diessel, 2007). So why do our data not reveal such a correlation? First, as discussed above, the effect of frequency on the

360 relationship between both $R_{0}$ estimates show that frequency affects diachronic stability

361 negatively among late acquired items, but does not do so among early acquired items. Since

362 Pagel et al. (2007) focused exclusively on core vocabulary (200 lexical core items), which is acquired early, they would not have seen the destabilizing effects of frequency on late acquired items. Lieberman et al. (2007) analyze the loss of 177 irregular verbal forms and find that their stability is positively correlated with frequency. The divergence between their result and ours is noteworthy. We suspect that it reflects that the frequencies employed in Lieberman et al. (2007) were derived from contemporary data (CELEX) rather than historically layered sources: in the slice representing most recent periods in Figure 2b (right), a negative interaction between stability and frequency is not visible either. We think that averaged frequencies, which cover the entire observation period, provide a more robust picture. ${ }^{10}$

Alternatively, there might be fundamental differences between phonotactics and the lexical domain. In the sublexical domain, the destabilizing effect of frequency might be stronger than in the lexical domain, because for the recognition of lexical items listeners can rely on the syntactic, semantic and pragmatic context, and may therefore recognize them even in phonetically reduced forms (Ernestus, 2014). In this regard, cluster perception is supported at best by morphological cues and benefits much less from linguistic redundancy. Therefore,

\footnotetext{
${ }^{10} \mathrm{We}$ would like to thank an anonymous reviewer for raising this issue.
} 
weakly entrenched phonotactic items may be more vulnerable to the destabilizing effects of frequency than weakly entrenched lexical items.

In summary, it appears that linguistic entrenchment is a function of both age of acquisition and frequency rather than just the latter (Ellis, 2012; Schmid, 2016). If we operationalize entrenchment by means of diachronic stability (because of the conserving function of routinization) then our analysis suggests that the relative age at which an items is acquired plays a key role in linguistic entrenchment. One straightforward mechanistic explanation is this: an item that happens to be acquired early has more time for being routinized than an item that is acquired late. Crucially, this holds irrespectively of how

387 frequent an item is. Another mechanism discussed by Monaghan (2014: 533), applies to the

388 lexical domain and involves higher plasticity of the cognitive system at early ages. Lexical items that are acquired early (for whatever reason) are more easily entrenched because the cognitive system is still more flexible. This, then, should also apply to complex processes of cognitive planning, articulation and perception relevant in the sublexical domain (Cholin, Dell, \& Levelt, 2011; Levelt \& Wheeldon, 1994). ${ }^{11}$ sets, sheds light on the question how much acquisition contributes to language change. To see this, note that the ratios derived from AoA data are considerably larger than the ones derived

${ }^{11}$ According to Nowak (2000), there is a third factor that influences the spread of items, namely network density. It is reflected in the number of users to which a learner is exposed. Thus, changes in the number of communicative contacts could cause socially motivated change in phonotactics (Trudgill (2001)), because $R_{0}$ decreases as the social network gets sparse. This relates to studies about the relationship between social structure and linguistic evolution (e.g. Wichmann, Stauffer, Schulze, and Holman (2008); Nettle (2012)), but based on the data that we analyzed in this study we cannot add to this discussion at this point. 
396 from diachronic data (Fig. 2, boxplots). While that difference may partly be an artefact of our

397 method $^{12}$, it may also be revealing. Thus, it might plausibly be interpreted as reflecting the

398 different contributions which first-language learners and proficient speakers make to the

399 actuation of linguistic change (Bybee, 2010; Croft, 2000). Since age-of-acquisition data

400 predict greater diachronic stability than is derivable from actual diachronic evidence, this

401 potentially suggests that language use by adults may play a more important role in causing

402 linguistic innovation than language acquisition by new generations of children (Diessel,

403 2012). Of course, further research is still needed to corroborate this suspicion, but the

404 methods we have demonstrated in this paper may help to make the question addressable in

405 quantitative terms.

12 To some extent, the difference may reflect the way in which $R_{0}^{\mathrm{GR}}$ has been estimated, because linguistic tokens and speakers represent two different dimensions in the first place. We suppose our token-frequency based proxy $r_{\mathrm{lg}}$ to represent a lower bound for the intrinsic growth rate $\rho$ in the population-dynamical model. This is because the spread of an item in a population of tokens involves both its spread through a population of speakers (i.e. $\rho$ ), and its spread through the linguistic system and the lexicon (Kroch (1989); Croft (2000); Denison (2003); Wang and Minett (2005); Blythe and Croft (2012)). The two dimensions are hard to disentangle on the basis of the limited number of historical texts available. Only quantitative empirical and computational approaches that incorporate both dimensions can shed more light on this issue.

As to $R_{0}^{\mathrm{AoA}}$, one possible reason why it might be overestimated is that our measure of AoA is based on lexical acquisition. Of course, the first form of a word that a child uses may not be the one containing the relevant cluster, nor will a child's first productions of what is a cluster in the target form always be accurate. Moreover, considering only AoA for estimating $R_{0}$ neglects the possibility that clusters, once acquired, may disappear again in adult speech - not only through language attrition and articulatory loss (see Seliger and Vago (1991); Ballard, Robin, Woodworth, and Zimba (2001); Torre and Barlow (2009)), but also through natural phonological backgrounding and deletion processes. If the proportion of individuals abandoning a particular cluster is underestimated, this will result in $R_{0}^{\text {AoA }}$ being overestimated. 


\section{Outlook}

407 Although our case study has been restricted to a very specific set of phonotactic constituents

408 and to a single language, namely English, there is no a priori reason why our approach

409 should not work in other domains (e.g. modeling the spread of single phonemes or words),

410 and for other languages. The two operationalizations of $R_{0}$, however, require (a) diachronic

411 data that cover the complete histories of constituents (ideally from the period of their first

412 emergence), as well as (b) corresponding acquisition data. As so often, English enjoys a

413 privileged status in this regard. A large number of historical sources have been digitized, and

414 also research on acquisition has produced a large amount of data. Testing the methods

415 described in this study against other languages is likely to face difficulties, although it would

416 of course be important. At least on the lexical level, however, the prospects are not so bad.

417 For core-vocabulary items in 25 languages a set of AoA ratings has been compiled by

418 Łuniewska et al. (2016), and diachronic resources such as the Google Books Ngram Corpus,

419 currently featuring eight languages, may serve as good starting points.

420

421 Acknowledgements: This work has been supported by FWF (grant no. P27592-G18).

422

423 Appendix

424 Table A1. Derived scores for each English type of final CC cluster used in empirical analysis:

425 logistic growth rate $r_{\mathrm{lg}}(2.2)$; goodness-of-fit measure $\mathrm{P}_{\mathrm{sp}}(2.2)$; basic reproductive ratio

426 estimated from logistic growth $R_{0}^{\mathrm{GR}}$ (2.2); age-of-acquisition AoA (2.3); basic reproductive

427 ratio estimated from AoA $R_{0}^{\text {AoA }}$ (2.3); total per million normalized frequency across all 
428 periods $\Sigma=18 \times\langle$ frequency $\rangle$ (2.4); average frequency across all periods $\langle$ frequency $\rangle$;

429 morphological status (2.5).

\begin{tabular}{|c|c|c|c|c|c|c|c|c|}
\hline cluster & AoA & $R_{0}^{\mathrm{AoA}}$ & $r_{\mathrm{lg}}$ & $\mathrm{P}_{\mathrm{sp}}$ & $R_{0}^{\mathrm{GR}}$ & $\Sigma$ & $\langle$ frequency & morph \\
\hline $\mathrm{bd}$ & 5.51 & 10.88 & 0.0083 & 0.86 & 1.25 & 2875.39 & 159.74 & illegal \\
\hline bz & 3.9 & 15.38 & 0.0089 & 0.83 & 1.27 & 3577.02 & 198.72 & illegal \\
\hline$d$ & 4.23 & 14.18 & 0.0066 & 0.76 & 1.2 & 1035.56 & 57.53 & illegal \\
\hline$d$ & 11.7 & 5.13 & 0.0081 & 0.77 & 1.24 & 182.59 & 10.14 & mixed \\
\hline $\mathrm{dz}$ & 2.91 & 20.64 & 0.0111 & 0.83 & 1.33 & 16066.49 & 892.58 & illegal \\
\hline d3 & 4.17 & 14.38 & 0.0024 & 0.86 & 1.07 & 17120.47 & 951.14 & legal \\
\hline z & 3.6 & 16.67 & 0.0137 & 0.86 & 1.41 & 624.26 & 34.68 & illegal \\
\hline fs & 3.98 & 15.08 & 0.0046 & 0.7 & 1.14 & 4236.11 & 235.34 & illegal \\
\hline $\mathrm{ft}$ & 3.96 & 15.14 & -0.001 & -0.16 & 0.97 & 18692.94 & 1038.5 & mixed \\
\hline gd & 3.06 & 19.63 & 0.0069 & 0.8 & 1.21 & 2462.6 & 136.81 & illegal \\
\hline gz & 2.79 & 21.48 & 0.0113 & 0.83 & 1.34 & 5024.83 & 279.16 & illegal \\
\hline ks & 2.89 & 20.79 & 0.0044 & 0.86 & 1.13 & 47399.45 & 2633.3 & mixed \\
\hline kt & 2.91 & 20.64 & 0.0118 & 0.93 & 1.35 & 33376.3 & 1854.24 & mixed \\
\hline $\mathrm{lb}$ & 6.74 & 8.9 & 0.0049 & 0.75 & 1.15 & 156.01 & 8.67 & legal \\
\hline Id & 3.23 & 18.58 & 0.0007 & 0.47 & 1.02 & 127823.96 & 7101.33 & mixed \\
\hline If & 4.21 & 14.25 & -0.0011 & -0.27 & 0.97 & 21867.05 & 1214.84 & legal \\
\hline $\mathrm{Ik}$ & 5.94 & 10.11 & -0.0025 & -0.84 & 0.92 & 10516.45 & 584.25 & legal \\
\hline $\mathrm{Im}$ & 8.26 & 7.27 & -0.0001 & 0.12 & 1 & 4858.57 & 269.92 & legal \\
\hline Ip & 5.87 & 10.22 & -0.0007 & -0.16 & 0.98 & 4273.8 & 237.43 & legal \\
\hline Is & 6.53 & 9.19 & -0.002 & -0.56 & 0.94 & 25955.21 & 1441.96 & mixed \\
\hline It & 4.3 & 13.94 & -0.0003 & 0.12 & 0.99 & 18907.59 & 1050.42 & mixed \\
\hline 1 & 7.92 & 7.57 & -0.0011 & -0.64 & 0.97 & 8198.53 & 455.47 & legal \\
\hline $\mathrm{Iz}$ & 3 & 19.98 & 0.0108 & 0.84 & 1.32 & 40839.21 & 2268.85 & illegal \\
\hline $\mathrm{md}$ & 3.87 & 15.5 & 0.0057 & 0.81 & 1.17 & 12894.59 & 716.37 & illegal \\
\hline $\mathrm{mf}$ & 9.21 & 6.51 & 0.0066 & 0.86 & 1.2 & 581.9 & 32.33 & legal \\
\hline $\mathrm{mp}$ & 3.73 & 16.09 & 0.0065 & 0.66 & 1.19 & 4675.2 & 259.73 & legal \\
\hline $\mathrm{mz}$ & 2.85 & 21.08 & 0.0035 & 0.81 & 1.11 & 22968.2 & 1276.01 & illegal \\
\hline nd & 3.19 & 18.81 & -0.0021 & -0.35 & 0.94 & 623823.11 & 34656.84 & mixed \\
\hline$d$ & 4.33 & 13.86 & 0.0062 & 0.84 & 1.19 & 1339.24 & 74.4 & illegal \\
\hline$k$ & 3.58 & 16.78 & 0.0086 & 0.86 & 1.26 & 10257.91 & 569.88 & legal \\
\hline ns & 4.63 & 12.95 & 0.001 & 0.21 & 1.03 & 94903.51 & 5272.42 & legal \\
\hline $\mathrm{nt}$ & 3.26 & 18.4 & 0.0036 & 0.97 & 1.11 & 133291.44 & 7405.08 & mixed \\
\hline $\mathrm{n}$ & 5.7 & 10.52 & -0.0011 & -0.8 & 0.97 & 6894.34 & 383.02 & mixed \\
\hline $\mathrm{nz}$ & 2.91 & 20.64 & 0.0138 & 0.83 & 1.41 & 71827.44 & 3990.41 & illegal \\
\hline$z$ & 3.88 & 15.48 & 0.0141 & 0.84 & 1.42 & 12585.83 & 699.21 & illegal \\
\hline ps & 2.74 & 21.92 & 0.0073 & 0.94 & 1.22 & 16989.12 & 943.84 & mixed \\
\hline pt & 2.74 & 21.92 & 0.0085 & 0.95 & 1.25 & 15427.24 & 857.07 & mixed \\
\hline
\end{tabular}




\begin{tabular}{|c|c|c|c|c|c|c|c|c|}
\hline $\mathrm{rb}$ & 8.1 & 7.41 & 0.0047 & 0.71 & 1.14 & 773.34 & 42.96 & legal \\
\hline rd & 3.35 & 17.89 & -0.0011 & -0.59 & 0.97 & 115745.44 & 6430.3 & mixed \\
\hline$r f$ & 7.04 & 8.53 & 0.0058 & 0.79 & 1.17 & 402.81 & 22.38 & legal \\
\hline rk & 3.95 & 15.2 & 0.0009 & 0.27 & 1.03 & 11891.15 & 660.62 & legal \\
\hline $\mathrm{rm}$ & 3.85 & 15.58 & 0.0025 & 0.89 & 1.08 & 9209.52 & 511.64 & legal \\
\hline $\mathrm{rn}$ & 4.08 & 14.69 & -0.0025 & -0.54 & 0.93 & 23164.88 & 1286.94 & legal \\
\hline $\mathrm{rp}$ & 7.41 & 8.09 & 0.0013 & 0.29 & 1.04 & 1957.53 & 108.75 & legal \\
\hline rs & 5.61 & 10.7 & -0.0002 & -0.28 & 1 & 51490.02 & 2860.56 & legal \\
\hline$r$ & 6.13 & 9.78 & -0.0037 & -0.91 & 0.89 & 20723.15 & 1151.29 & mixed \\
\hline$r z$ & 3.11 & 19.29 & 0.0125 & 0.83 & 1.38 & 23445.87 & 1302.55 & illegal \\
\hline sk & 4.42 & 13.58 & 0.0065 & 0.96 & 1.2 & 4500.53 & 250.03 & legal \\
\hline $\mathrm{sp}$ & 6.95 & 8.63 & 0.0063 & 0.76 & 1.19 & 860.12 & 47.78 & legal \\
\hline st & 2.69 & 22.28 & 0.0017 & 0.75 & 1.05 & 164960.88 & 9164.49 & mixed \\
\hline $\mathrm{t}$ & 3.73 & 16.09 & 0.0078 & 0.95 & 1.24 & 14280.96 & 793.39 & illegal \\
\hline ts & 2.9 & 20.71 & 0.0062 & 0.92 & 1.18 & 71384.23 & 3965.79 & mixed \\
\hline $\mathrm{t}$ & 4.24 & 14.16 & -0.004 & -0.6 & 0.88 & 96962.87 & 5386.83 & legal \\
\hline$S$ & 4.32 & 13.9 & 0.0026 & 0.4 & 1.08 & 62.73 & 3.49 & illegal \\
\hline tz & 8.85 & 6.78 & 0.0064 & 0.76 & 1.19 & 90.09 & 5 & illegal \\
\hline $\mathrm{zd}$ & 3.43 & 17.51 & 0.0093 & 0.94 & 1.28 & 22371.96 & 1242.89 & illegal \\
\hline $3 d$ & 5.51 & 10.9 & 0.0093 & 0.92 & 1.28 & 6219.11 & 345.51 & illegal \\
\hline $\mathrm{zm}$ & 11.66 & 5.14 & 0.007 & 0.74 & 1.21 & 152.89 & 8.49 & legal \\
\hline
\end{tabular}

\section{$431 \quad$ References}

432 Anderson, R., \& May, R. (1991). Infectious diseases of humans: Dynamics and control. New 433 York: Oxford University Press.

434 Ballard, K. J., Robin, D., Woodworth, G., \& Zimba, L. (2001). Age-related changes in motor 435 control during articulator visuomotor tracking. Journal of Speech, Language, and Hearing $436 \quad$ Research, 44, 763-777.

437 Berent, I., Steriade, D., Lennertz, T., \& Vaknin, V. (2007). What we know about what we 438 have never heard: Evidence from perceptual illusions. Cognition, 104(3), 591-630. https://doi.org/10.1016/j.cognition.2006.05.015 
440 Blythe, R. A., \& Croft, W. (2012). S-curves and the mechanism of propagation in language $441 \quad$ change. Language, 88(2), 269-304.

442 Briscoe, T. (Ed.). (2008). Linguistic Evolution through Language Acquisition. Cambridge: 443 Cambridge University Press.

444 Brown, R. (1973). A first language: The early stages. Harvard: Harvard University Press.

445 Bybee, J. (2007). Frequency of use and the organization of language. Oxford: Oxford $446 \quad$ University Press.

447 Bybee, J. (2010). Language, Usage and Cognition. Cambridge: Cambridge University Press.

448 Carnegie Mellon Speech Group. (2014). Carnegie Mellon Pronouncing Dictionary: Carnegie 449 Mellon University. Retrieved from http://www.speech.cs.cmu.edu/cgi-bin/cmudict

450 Cavalli-Sforza, L. L., \& Feldman, M. W. (1981). Cultural transmission and evolution: 451 aquantitative approach. Princeton: Princeton University Press.

452 Cholin, J., Dell, G. S., \& Levelt, W. J. M. (2011). Planning and articulation in incremental 453 word production: syllable-frequency effects in English. Journal of experimental 454 psychology. Learning, memory, and cognition, 37(1), 109-122. 455 https://doi.org/10.1037/a0021322

456 Croft, W. (2000). Explaining language change: An evolutionary approach. Longman 457 linguistics library. Harlow, England, New York: Longman.

458 Davies, M. (2008). The corpus of contemporary American English (COCA): 450 million $459 \quad$ words, 1990-2012: Brigham Young University.

460 Davies, M. (2010). The corpus of historical American English: COHA. Brigham: BYE, $461 \quad$ Brigham Young University. 
462 Dawkins, R. (1976). The selfish gene. New York, Oxford: Oxford University Press.

463 Denison, D. (2003). Log(ist)ic and simplistic S-curves. In R. Hickey (Ed.), Motives for 464 Language Change (pp. 54-70). Cambridge: Cambridge University Press.

465 Diessel, H. (2007). Frequency effects in language acquisition, language use, and diachronic $466 \quad$ change. New ideas in psychology. (25), 108-127.

467 Diessel, H. (2012). New Perspectives, Theories and Methods: Diachronic change and 468 language acquisition. In A. Bergs \& L. J. Brinton (Eds.), Historical linguistics of English 469 (pp. 1599-1613). Berlin [a.e.]: De Gruyter Mouton.

470 Dietz, K. (1993). The estimation of the basic reproduction number for infectious diseases. $471 \quad$ Statistical methods in medical research, 2, 23-41.

472 Dressler, W. U., Dziubalska-Kołaczyk, K., \& Pestal, L. (2010). Change and variation in 473 morphonotactics. Folia Linguistica

Historica,

31 $51-68$. https://doi.org/10.1515/flih.2010.003

475

Ellis, N. C. (2012). What can we count in language, and what counts in language acquisition, 476 cognition, and use? In S. T. Gries \&

D. Divjak (Eds.), Frequency Effects in Language 477 Learning and Processing. Berlin, Boston: De Gruyter. https://doi.org/10.1515/9783110274059.7

479 Ernestus, M. (2014). Acoustic reduction and the roles of abstractions and exemplars in $480 \quad$ speech processing. Lingua, 142, 27-41.

481 Hay, J., \& Baayen, H. (2005). Probabilistic phonotactics and morphological productivity. 482 Retrieved from http://www.ling.canterbury.ac.nz/jen/documents/haybaayenh_esse.htm 
483 Heffernan, J., Smith, R., \& Wahl, L. (2005). Perspectives on the basic reproductive ratio. 484 Journal of The Royal Society Interface, 2(4), 281-293. 485 https://doi.org/10.1098/rsif.2005.0042

486 Henrich, J., \& Boyd, R. (2002). On Modeling Cognition and Culture: Why cultural evolution 487 does not require replication of representations. Journal of Cognition and Culture, 2(2), 87-112. https://doi.org/10.1163/156853702320281836

489 Hethcote, H. (1989). Three basic epidemiological models. In S. Levin, T. Hallam, \& L. Gross $490 \quad$ (Eds.), Biomathematics: Vol. 18. Applied Mathematical Ecology.

491 Korecky-Kröll, K., Dressler, W. U., Freiberger, E. M., Reinisch, E., Mörth, K., \& Libben, G. 492 (2014). Morphonotactic and phonotactic processing in German-speaking adults. Language $493 \quad$ Sciences, 46, 48-58.

494 Kroch, A. (1989). Reflexes of Grammar in Patterns of Language Change. Language 495 Variation and Change, 1, 199-244.

Kroch, A., Santorini, B., \& Delfs, L. (2004). Penn-Helsinki Parsed Corpus of Early Modern English. Retrieved from http://www.ling.upenn.edu/hist-corpora/

Kroch, A., Santorini, B., \& Diertani, A. (2016). The Penn Parsed Corpus of Modern British English (PPCMBE2): Department of Linguistics, University of Pennsylvania. Retrieved from http://www.ling.upenn.edu/ppche/ppche-release-2016/PPCMBE2-RELEASE-1

501 Kroch, A., \& Taylor, A. (2000). Penn-Helsinki Parsed Corpus of Middle English. Retrieved 502 from http://www.ling.upenn.edu/hist-corpora/

503 Kuperman, V., Stadthagen-Gonzalez, H., \& Brysbaert, M. (2012). Age-of-acquisition ratings 504 for 30,000 English words. Behavior Research Methods, 44, 978-990. 
505 Kuperman, V., Ernestus, M., \& Baayen, H. (2008). Frequency distributions of uniphones, 506 diphones, and triphones in spontaneous speech. The Journal of the Acoustical Society of $507 \quad$ America, 124, 3897-3908.

508 Lancaster, H. O. (1990). Expectations of Life: A Study in the Demography, Statistics, and $509 \quad$ History of World Mortality. New York, NY: Springer New York.

510 Levelt, W. J., \& Wheeldon, L. (1994). Do speakers have access to a mental syllabary? 511 Cognition, 50(1-3), 239-269. https://doi.org/10.1016/0010-0277(94)90030-2

512 Lieberman, E., Michel, J.-B., Jackson, J., Tang, T., \& Nowak, M. A. (2007). Quantifying the 513 evolutionary dynamics of language. Nature, 449(7163), 713-716. 514 https://doi.org/10.1038/nature06137

515 Łuniewska, M., Haman, E., Armon-Lotem, S., Etenkowski, B., Southwood, F., Anđelković, 516 D.,. . Ünal-Logacev, Ö. (2016). Ratings of age of acquisition of 299 words across 25 517 languages: Is there a cross-linguistic order of words? Behavior Research Methods, 48(3), 1154-1177. https://doi.org/10.3758/s13428-015-0636-6

519 MacNeilage, P. F., \& Davis, B. L. (2000). On the origin of internal structure of word forms. $520 \quad$ Science, $288,527-531$.

521 McQueen, J. (1998). Segmentation of Continuous Speech Using Phonotactics. Journal of $522 \quad$ Memory and Language, 39, 21-46.

523 Mesgarani, N., Cheung, C., Johnson, K., \& Chang, E. F. (2014). Phonetic feature encoding in 524 human superior temporal gyrus. Science, 343, 1006-1010.

525 Minkova, D. (1991). The history of final vowels in English: The sound of muting. Topics in $526 \quad$ English linguistics: Vol. 4. Berlin, New York: M. de Gruyter. 
527 Monaghan, P. (2014). Age of acquisition predicts rate of lexical evolution. Cognition, 133, $528 \quad 530-534$.

529 Nettle, D. (2012). Social scale and structural complexity in human languages. Phil. Trans. $R$. $530 \quad$ Soc. B, 367, 1829-1836.

531 Newberry, M. G., Ahern, C. A., Clark, R., \& Plotkin, J. B. (2017). Detecting evolutionary 532 forces in language change. Nature, 551(7679), 223-226. 533 https://doi.org/10.1038/nature24455

534 Niyogi, P. (2006). The computational nature of language learning and evolution. Cambridge, 535 MA.

536 Nowak, M. A. (2000). The basic reproductive ratio of a word, the maximum size of a lexicon. Journal of theoretical biology, 204(2), 179-189. https://doi.org/10.1006/jtbi.2000.1085

Nowak, M. A., Plotkin, J., \& Jansen, V. (2000). The evolution of syntactic communication. Nature, 404(6777), 495-498.

540 Reali, F., \& Griffiths, T. D. (2009). The evolution of frequency distributions: Relating 541 regularization to inductive biases through iterated learning. Cognition, 111, 317-328.

542 Schmid, H.-J. (2016). Linguistic entrenchment and its psychological foundations. In H.-J. 543 Schmid (Ed.), Entrenchment and the psychology of language learning (pp. 435-452). 544 Berlin: Mouton de Gruyter.

545 Seliger, H., \& Vago, R. (Eds.). (1991). First Language Attrition. Cambridge: CUP.

546 Smith, K., \& Kirby, S. (2008). Cultural evolution: implications for understanding the human 547 language faculty and its evolution. Philosophical transactions of the Royal Society of 548 London. Series B, Biological sciences, 363(1509), 3591-3603. 549 https://doi.org/10.1098/rstb.2008.0145 
550 Smith, K., Perfors, A., Fehér, O., Samara, A., Swoboda, K., \& Wonnacott, E. (2017).

551 Language learning, language use, and the evolution of linguistic variation. Philosophical 552 Transactions of the Royal Society B. Advance online publication. 553 https://doi.org/10.1098/rstb.2016.0051

554 Smith, K., \& Wonnacott, E. (2010). Eliminating unpredictable variation through iterated learning. Cognition, 116, 444-449.

556 Solé, R. V. (2011). Phase transitions. Princeton: Princeton University Press.

557 Solé, R. V., Corominas-Murtra, B., \& Fortuny, J. (2010). Diversity, competition, extinction: 558 the ecophysics of language change. Journal of The Royal Society Interface, 7(53), 16471664.

Retrieved from http://groups.lis.illinois.edu/amag/langev/paper/sole2010diversitygsc.html

Sperber, D. (1985). Anthropology and psychology: Towards an epidemiology of representations. Man, 73-89.

Szmrecsanyi, B. (2012). Analyticity and syntheticity in the history of English. In T. (pp. 654-665). Oxford: Oxford University Press.

566 Torre, P., \& Barlow, J. A. (2009). Age-related changes in acoustic characteristics of adult speech. Journal of Communication Disorders, 42(5), 324-333.

568 Trudgill, P. (2001). Sociolinguistic variation and change. Edinburgh: Edinburgh University $569 \quad$ Press.

570 Wang, W., \& Minett, J. (2005). The invasion of language: Emergence, change and death. Trends in Ecology and Evolution, 20, 263-269. 
572 Wichmann, S., Stauffer, D., Schulze, C., \& Holman, E. (2008). Do language change rates 573 depend on population size? Advances in Complex Systems, 11(3), 357-369.

574 Wood, S. (2006a). Generalized Additive Models: an introduction with R. Boca Raton, 575 Florida: Chapman \& Hall/CRC.

576 Wood, S. (2006b). Low-Rank Scale-Invariant Tensor Product Smooths for Generalized 577 Additive Mixed Models. Biometrics, 62, 1025-1036. https://doi.org/10.1111/j.1541$578 \quad 0420.2006 .00574 . x$

579 Worden, R. (2008). Linguistic structure and the evolution ofwords. In T. Briscoe (Ed.), $580 \quad$ Linguistic Evolution through Language Acquisition (pp. 75-100). Cambridge: Cambridge $581 \quad$ University Press. 